\title{
Drug transporter regulation in tumors by DNA methylation
}

\author{
Oliver Zolk and Martin F Fromm* \\ See research article http://www.genomemedicine.com/content/3/12/82
}

\begin{abstract}
Epigenetic alterations, such as aberrant DNA methylation, are a hallmark of cancer. DNA

hypermethylation of the promoter region affects, for example, the expression of tumor suppressor genes and is associated with their transcriptional silencing in tumors. A recent report has provided evidence for epigenetic silencing of the multispecific organic cation transporter SLC22A1 in hepatocellular carcinoma. Given the role of this transporter in the cellular uptake of several anticancer drugs, the study provided a novel mechanism to explain the substantial variability in treatment response, and it might provide a new strategy for optimization of pharmacotherapy of hepatocellular carcinoma.
\end{abstract}

Development of hepatocellular carcinoma, in common with other types of tumor, is considered to be a multistep process. Genetic and epigenetic alterations accumulate in regulatory genes, leading to activation of oncogenes and inactivation or loss of tumor suppressor genes [1]. The concept that hepatocellular carcinoma is a disease of epigenetic as well as genetic alterations has been validated in the past two decades. The epigenetic pathway is, in contrast to the genetic events, a reversible alteration that is not caused by primary DNA sequence changes.

There are three main epigenetic mechanisms: (i) hypermethylation of $\mathrm{CpG}$ islands in promoter sequences leading to silencing of tumor suppressor genes; (ii) DNA hypomethylation, which causes genomic instability or induction of genes involved, for example, in cell growth and invasion [2]; and (iii) histone modification, which affects

*Correspondence: fromm@pharmakologie.med.uni-erlangen.de Institute of Experimental and Clinical Pharmacology and Toxicology, FriedrichAlexander-Universität Erlangen-Nürnberg, Fahrstrasse 17, 91054 Erlangen, Germany chromatin conformation. Because epigenetic mechanisms may function as an interface between environmental factors and the genome [3], deregulation of the epigenome by environmental stressors (for example, hepatitis $\mathrm{B}$ and hepatitis $\mathrm{C}$ viruses, chronic alcohol intake, and aflatoxins) is believed to disrupt cellular processes and contribute to the risk of hepatocellular carcinoma. The early appearance of epigenetic changes makes them attractive targets for biomarker discovery. Moreover, drugs to reverse the epigenetic abnormalities are under development and some have already been approved. Cancer epigenetics is continuously translating into clinical practice and will help to optimize cancer diagnostics and treatment. The recent observation by Schaeffeler et al. in Genome Medicine that uptake transporters for anticancer drugs are epigenetically regulated in hepatocellular carcinoma adds an important piece of information to the growing body of research on cancer epigenetics [4]. The potential implications of these findings for biomarker development and pharmacotherapy of hepatocellular carcinoma will be discussed.

\section{Epigenetics of organic cation transporter genes in cancer}

Whereas previous work has focused mainly on the role of CpG hypermethylation for regulating oncogenes, tumor suppressor genes or DNA repair genes in hepatocellular carcinoma, Schaeffeler et al. [4] investigated the role of DNA methylation in the expression of specific members of the solute carrier (SLC) gene superfamily, namely the organic cation transporter genes SLC22A1 to SLC22A3 (encoding proteins OCT1, OCT2 and OCT3, respectively). In normal hepatocytes, SLC22A1 (solute carrier family 22 member 1), which encodes OCT1 (organic cation transporter 1), is highly expressed and is responsible for the uptake of nutrients, metabolites and xenobiotics. The transporter is also crucial for the uptake of some drugs [5]. Whereas lipophilic antineoplastic agents can enter cells by passive diffusion, uptake of charged hydrophilic agents, such as the anticancer compounds imatinib, cisplatin, oxaliplatin, picoplatin, irinotecan and 
paclitaxel, requires active transport. Indeed, OCT1 transports these six drugs and thereby contributes to the susceptibility of cancer cells to these antineoplastic drugs.

cDNA microarray studies have demonstrated decreased expression of SLC22A1 in hepatocellular carcinoma compared with normal liver tissue. However, the mechanisms of downregulation have remained unclear. Based on their findings, Scheffeler et al. [4] suggest SLC22A1 promoter hypermethylation as an important mechanism. Epigenetic silencing of OCT1 in hepatocellular carcinoma, as demonstrated by Schaeffeler et al. [4], most likely impairs uptake of some anticancer drugs into hepatocellular cancer cells and thus is likely to impair their efficacy. This might, in part, explain the low response rates and uncertain survival benefit of, for example, cisplatin-based chemotherapy for hepatocellular carcinoma. In contrast, the tyrosine kinase inhibitor sorafenib, which does not appear to require active transport to enter cells [6], induces better disease control, significantly extends survival, and has become a standard first-line option for systemic treatment of advanced hepatocellular carcinoma.

\section{Biomarkers to guide individualization of chemotherapy}

Given the important role of OCT1 in cancer cell uptake and thus treatment efficacy of several anticancer drugs, methylation of SLC22A1 might be used as a marker for predicting therapeutic response. The work by Schaeffeler et al. [4] has demonstrated substantial interindividual variability in the degree of SLC22A1 methylation and SLC22A1 production, as revealed by histochemical analysis. It is tempting to speculate that patients with a high SLC22A1 methylation phenotype and silenced transporter expression may exhibit chemoresistance, whereas patients with a low methylation phenotype and enhanced transporter expression may respond to chemotherapy. Most recently, Ma et al. [7] provided another example of the involvement of epigenetic mechanisms in determining chemoresistance and chemosensitivity in hepatocellular carcinoma. MIR193A promoter hypomethylation is associated with transcriptional induction of the microRNA 193a-3p, which targets the serine/ arginine-rich splicing factor 2 (SRSF2). SRSF2 sensitizes hepatocellular cancer cells to the chemotherapeutic drug 5-fluorouracil via upregulation of the proapoptotic splicing form of caspase 2. Accordingly, its repression by microRNA 193a-3p reduces sensitivity of hepatocellular cancer cells to 5-fluorouracil [7]. This experimental observation suggests that the DNA methylation state of MIR193A could function as a marker to predict the therapeutic response to 5 -fluorouracil.

\section{Biomarker for cancer screening}

As well as using DNA methylation as a biomarker for personalizing chemotherapy, evidence has emerged that detection of abnormal promoter $\mathrm{CpG}$ island hypermethylation is also a potential biomarker for risk of developing cancer. DNA methylation in adjacent histologically normal liver tissue of hepatocellular carcinoma has been reported from several groups and is now accepted as an early event in cancer development [8]. Thus, aberrant DNA methylation might be useful for early cancer detection and for predicting prognosis. Comparisons of $\mathrm{CpG}$ island methylation in hepatocellular carcinoma with that in adjacent tumor-free tissue or normal control livers have produced a continuously expanding list of potential marker genes. Interestingly, Schaeffeler et al. [4] demonstrated that SLC22A1 belongs to a group of genes with progressively increasing $\mathrm{CpG}$ methylation, from normal liver to hepatocellular carcinoma, with intermediate methylation in precancerous tissues (that is, in adjacent tumor-free tissue). Similar methylation changes related to the progression of malignant transformation have been observed previously for other genes, including RASSF1A, PRDM14 and TBX4, which encode Ras association domain family member 1 , PR domain containing 14 and T-box 4, respectively. [8]. Therefore, the CpG methylation phenotype of these genes may serve as a marker for early cancer detection or assessing cancer risk.

As biomarker strategies move towards actual clinical practice, these proof-of-principle findings should be validated in larger patient cohorts. For detection of other tumors, such as colorectal and lung cancers, assays that test methylation patterns of marker genes in DNA from the stool, blood or bronchoalveolar lavage are already commercially offered to clinicians [9].

\section{Epigenetic therapy for cancer}

The results reported by Shaeffeler et al. [4] also highlight the clinical implications of epigenetic modulation of transporter expression. DNA methyltransferase inhibitors, such as decitabine, have already been approved for treatment of lymphomas and are being tested as a therapeutic option against various solid tumors. If these agents are concomitantly administered with classical anticancer drugs, the resulting induction of transporter expression may influence the disposition and effect of these anticancer drugs. Specifically, repression of OCT1 might be reversed by treatment with the DNA methyltransferase inhibitor decitabine, enhancing uptake of cisplatin into hepatocellular tumor cells. However, the fascinating possibility of overcoming the problem of chemoresistance with an epigenetic therapy awaits proof of concept. 


\section{Abbreviations}

OCT1, organic cation transporter 1; SRSF2, serine/arginine-rich splicing factor 2 .

\section{Competing interests}

The authors declare that they have no competing interests.

Published: 31 January 2012

\section{References}

1. Tischoff I, Tannapfe A: DNA methylation in hepatocellular carcinoma. World J Gastroenterol 2008, 14:1741-1748.

2. Stefanska B, Huang J, Bhattacharyya B, Suderman M, Hallett M, Han ZG, Szyf M: Definition of the landscape of promoter DNA hypomethylation in liver cancer. Cancer Res 2011, 71:5891-5903.

3. Herceg Z, Vaissiere T: Epigenetic mechanisms and cancer: an interface between the environment and the genome. Epigenetics 2011, 6:804-819.

4. Schaeffeler E, Hellerbrand C, Nies AT, Winter S, Kruck S, Hofmann U, van der Kuip H, Zanger UM, Koepsell H, Schwab M: DNA methylation is associated with downregulation of the organic cation transporter OCT1 (SLC22A1) in human hepatocellular carcinoma. Genome Med 2011, 3:82.

5. Zolk O, Fromm MF: Transporter-mediated drug uptake and efflux: important determinants of adverse drug reactions. Clin Pharmacol Ther 2011, 89:798-805.

6. Hu S, Chen Z, Franke R, Orwick S, Zhao M, Rudek MA, Sparreboom A, Baker SD: Interaction of the multikinase inhibitors sorafenib and sunitinib with solute carriers and ATP-binding cassette transporters. Clin Cancer Res 2009 15:6062-6069.

7. Ma K, He Y, Zhang H, Fei Q, Niu D, Wang D, Ding X, Xu H, Chen X, Zhu J: DNA methylation regulated miR-193a-3p dictates resistance of hepatocellular carcinoma to 5 -fluorouracil via SRSF2. J Biol Chem, in press.

8. Gao W, Kondo Y, Shen L, Shimizu Y, Sano T, Yamao K, Natsume A, Goto Y, Ito M, Murakami H, Osada H, Zhang J, Issa JP, Sekido Y: Variable DNA methylation patterns associated with progression of disease in hepatocellular carcinomas. Carcinogenesis 2008, 29:1901-1910.

doi:10.1186/gm309

Cite this article as: Zolk O, Fromm MF: Drug transporter regulation in tumors by DNA methylation. Genome Medicine 2012, 4:10. 
Zolk and Fromm Genome Medicine 2012, 4:10

Page 4 of 3

http://genomemedicine.com/content/4/1/10

9. Müller HM, Oberwalder M, Fiegl H, Morandell M, Goebel G, Zitt M, Muhlthaler M, Ofner D, Margreiter R, Widschwendter M: Methylation changes in faecal DNA: a marker for colorectal cancer screening? Lancet 2004, 363:1283-1285. 